\title{
Editorials
}

\section{New opportunities for anesthesia research in Canada}

\author{
Beverley A. Orser MD PhD, ${ }^{*}$ Donald R. Miller MD $†$
}

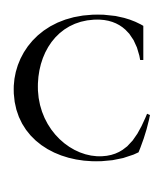

LINICAL innovation and scientific discovery are the hallmarks of every successful medical enterprise. The specialty of anesthesia is no exception. Anesthesiologists are currently leaders in the field of patient safety and perioperative care because of the creative endeavours of our predecessors. ${ }^{1}$ The dramatic reduction in mortality in patients undergoing anesthesia has been attributed, in part, to monitoring systems that evolved from anesthesia laboratories. Practice guidelines directly resulted from studies in patient outcome. Indeed, the first Guidelines to the Practice of Anesthesia published by the Canadian Anesthesiologists' Society (CAS) in 1973 are credited with improving patient care and reducing the soaring costs of malpractice premiums. ${ }^{2}$ No other specialty or anesthesia society had previously developed guidelines of this kind. Bench research has also made tremendous progress identifying specific proteins in the brain, particularly neurotransmitter receptors, as molecular targets for anesthetics. ${ }^{3}$ The clinical and scientific advances during the past 50 years have been unprecedented.

To remain at the cutting edge requires constant rejuvenation and renewal. We need to consider how colleagues in other disciplines view our current research efforts. Are we addressing problems that are important to medicine in general, as well as to the specialty? Are we applying the latest scientific discoveries and technology to anesthetic-related problems? The evidence presented below suggests we need to revise our research strategy. The current shortage of anesthesiologists and lack of resources for academic programs have severely challenged research productivity. Consequently, creative and targeted solutions are required to develop a cohort of young investigators who will lead the specialty.

\section{Indicators of decline in anesthesia research}

Several lines of evidence indicate a decline in anesthesia research, although this problem is not unique to Canada. The number of original articles submitted from Canada to the Canadian Journal of Anesthesia has gradually decreased over the past decade. South of the border, approximately, $60 \%$ of manuscripts received last year by the journal Anesthesiology, were sent from outside the United States. Japan, Germany, and France now lead the international submission rates, with Canada trailing behind. ${ }^{4}$ Enrolment in research training programs also indicates that anesthesiology lags behind other specialties (Figure). In 1995, The Royal College of Physicians and Surgeons of Canada established the Clinician Investigator Program (CIP) to facilitate the research training of clinicians (http://rcpsc.medical.org). This program provides an intense period of research training (a minimum of two years) for a small group of residents who are selected during their junior years of postgraduate clinical training. In 2001, only one graduate from the 16 Canadian University Residency programs took advantage of this tremendous opportunity. ${ }^{5}$ Also, the number of research grants awarded from the Canadian Institutes of Health Research (CIHR; http://www.cihr.ca) to faculty members of Canadian University Departments of Anesthesia is significantly below that of most other medical specialties. In spite of a massive increase in available CIHR funding opportunities, only a handful of anesthesiologists in Canada hold research grants from the CIHR, our primary national funding agency for health research.

\section{New funding opportunities in Canada}

Despite such gloomy indicators, there is substantial reason for optimism. First, support for anesthesiarelated research has become increasingly available in

\footnotetext{
From Departments of Anesthesia, University of Toronto* and The University of Ottawa, $†$ Canada. Address correspondence to: Dr. Beverley A. Orser, Department of Anesthesia, Sunnybrook and Women's College Health Sciences Centre, 2075 Bayview Avenue, Toronto, Ontario M4N 3N5, Canada; Phone: 416-480-4864; Fax: 416-978-4940;

E-mail: Beverley.orser@utoronto.ca
} 
CIP Trainees by specialty or subspecialty (2001)

\begin{tabular}{llll}
\hline & CIP trainees & $\begin{array}{l}\text { Number of } \\
\text { residents }\end{array}$ & $\%$ \\
\hline Surgery & 45 & 229 & 19.7 \\
Medicine & 28 & 282 & 9.9 \\
Pediatrics & 8 & 91 & 8.8 \\
Psychiatry & 6 & 108 & 5.6 \\
Ophthalmology & 4 & 21 & 19 \\
Community medicine & 4 & 18 & 22.2 \\
Clinical pharmacology & 2 & 2 & 100 \\
Radiation oncology & 1 & 10 & 10 \\
Obstetrics and gynecology & 1 & 42 & 2.4 \\
Anatomical pathology & 1 & 13 & 7.7 \\
Anesthesiology & 1 & 66 & 1.5 \\
\hline
\end{tabular}

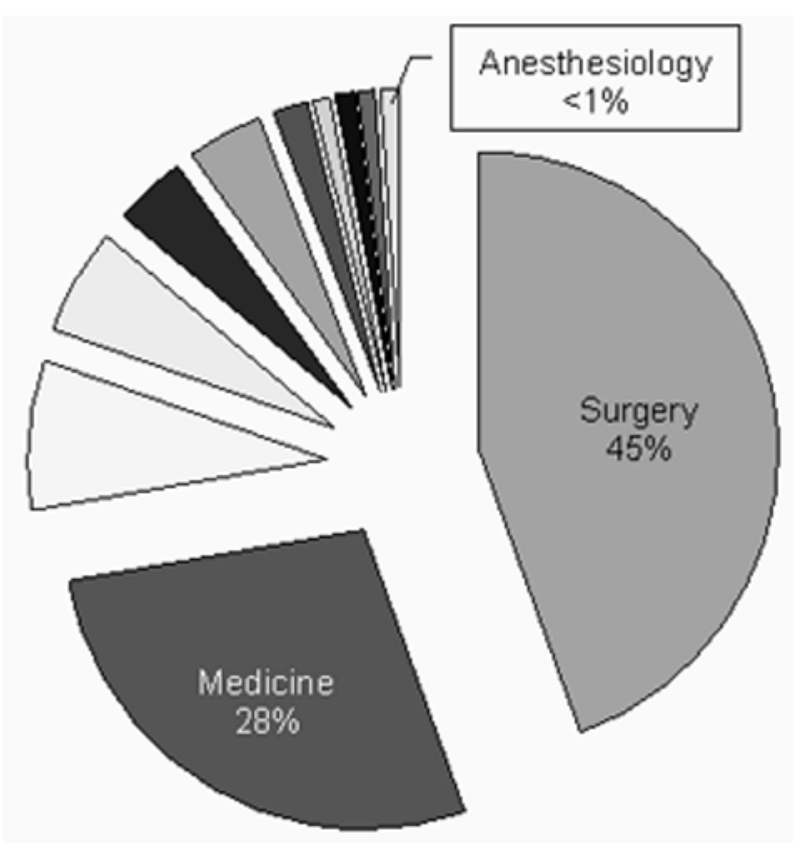

FIGURE The total number of Clinical Investigator Program (CIP) trainees in 2001 at the University of Toronto compared to the number of residents registered in the various medical and surgical disciplines as reported by the Royal College of Physicians and Surgeons of Canada. No additional CIP trainees were enrolled in other Canadian anesthesia residency programs.

Canada. Funding from the Canadian Anesthesia Research Foundation (CARF) has increased for the first time in five years due to a significant growth in capital. With additional increases from industry partners and the CIHR, funding opportunities available to CAS members have reached an all-time high. The CAS Research Grants and Fellowship Program now includes a Career Scientist Award valued at \$120,000, a Research Fellowship Award of $\$ 95,000$, and six research grants with a cumulative value of $\$ 120,000$. Moreover, these research opportunities recognize the different levels of experience of young and established investigators. The recent competition for the newlyestablished Career Scientist Award in Anesthesia was an immense success, with 13 high quality applications originating from four provinces. Clearly, there is widespread interest in research across the country.

In addition to these important opportunities, new partnerships between the CAS and CIHR are currently being explored to create a Clinician Scientists Career Pathway. The mandate of the CIHR is to facilitate knowledge transfer (bench-to-bedside research, and translational research). Acquisition of new medical knowledge is no longer sufficient; rather, the CIHR seeks innovative ways to accelerate the transfer of knowledge to improve health care. It is our view that anesthesiologists are well positioned to fulfill this role as trained physiologists, pharmacologists, and perioperative physicians. By focusing on our unique strengths and skills, we can develop competitive and productive research programs.

Finally, university departments have the opportunity to develop additional research resources. For example, endowed Research Chairs in Anesthesia generally provide salary support for established investigators. Although endowed chairs do require considerable capital (usually $\$ 2 \mathrm{M}$ ), several now exist including the Harold Griffith Chair at McGill University and the new Fraser Elliott Research Chair in Cardiovascular Anesthesia at the University of Toronto. Also, the Laws Fellowship Award at the University of Toronto provides one to two years of full salary support for anesthesia residents or junior fellows undertaking graduate studies. Hopefully, the next decade will witness the creation of additional comprehensive, stable funding sources within the specialty. In this context, alternate funding plans (AFPs) should provide important opportunities to increase protected research time. Currently, AFP's exist at several centres in British Columbia, Alberta and Ontario. A number of other academic health sciences centres are considering or negotiating AFPs; research should be given a high priority amongst the "deliverables" in these negotiations.

Most importantly, the building block of a successful research program is a well-trained investigator supported by skilled mentors and a committed University Chair. A brief period of research training during a fellowship year is unlikely to be adequate in today's competitive environment. Candidates successfully funded by CIHR generally have at least two years of research training. The quality of the training program cannot 
be underestimated. For most candidates, this involves completing a graduate degree outside their medical discipline. By selecting a limited number of committed residents or fellows, we too can increase this cohort within our specialty. The CAS meeting would seem an appropriate forum for CIP trainees and others to discuss their experience with potential recruits.

\section{Conclusions}

There is no one simple solution to enhancing research productivity within the specialty. However, within the past several years, increasing numbers of grants and research funding mechanisms have become available. The CIP program and restructuring of the CIHR provide an opportunity to revise our current models of training and support for investigators. Through our Academic Health Science Centres, the CAS, and provincial and national funding agencies, there are abundant opportunities for research in the specialty. Our challenge is clearly to seize these opportunities. In many respects, the future of anesthesia research Canada has never been better.

\section{Acknowledgements}

The authors thank Drs. David Bevan and Robert Byrick for their constructive comments about this editorial.

\section{Nouvelles possibilités de recherche en anesthésie au Canada}

L'innovation clinique et les découvertes scientifiques sont les deux signes majeurs du succès de toute entreprise médicale. La spécialité de l'anesthésie ne fait pas exception. Les anesthésiologistes sont présentement les chefs de file en matière de sécurité des patients et de soins périopératoires grâce aux efforts créatifs de leurs prédécesseurs. ${ }^{1}$ La réduction importante de la mortalité des patients qui subissent une anesthésie a été attribuée, en partie, aux systèmes de surveillance issus des laboratoires d'anesthésie. Les directives cliniques ont découlé directement des études de résultats cliniques. En effet, on attribue au premier Guide d'exercice de l'anesthésie publié par la Société canadienne des anesthésiologistes (SCA) en 1973 l'amélioration des soins et la réduction du coût élevé des primes d'assur- ance responsabilité. ${ }^{2}$ Aucune autre spécialité ou société d'anesthésie n'avait encore élaboré un guide semblable. La recherche avancée a aussi beaucoup évolué par la découverte de protéines spécifiques du cerveau, en particulier les récepteurs neurotransmetteurs, comme cibles moléculaires des anesthésiques. ${ }^{3}$ Les progrès cliniques et scientifiques réalisés pendant les 50 dernières années sont sans précédents.

Demeurer à la fine pointe exige un rajeunissement et un renouvellement constants. Nous devons considérer comment nos collègues d'autres disciplines voient nos efforts actuels dans le domaine de la recherche. Abordons-nous des problèmes importants pour la médecine en générale aussi bien que pour notre spécialité? Appliquons-nous les plus récentes technologies et découvertes scientifiques aux problèmes reliés à l'anesthésie? Les données qui suivent laissent croire que nous devons revoir notre stratégie de recherche. La pénurie actuelle d'anesthésiologistes et le manque de ressources pour les programmes universitaires ont sérieusement remis en cause la productivité en recherche. En conséquence, des solutions créatrices et ciblées sont nécessaires pour constituer une cohorte de jeunes chercheurs qui seront les porte-parole de la spécialité.

\section{Les indicateurs du déclin de la recherche en anesthésie}

Certaines sources de données laissent voir un déclin de la recherche en anesthésie, quoique ce problème ne soit pas unique au Canada. Le nombre d'articles originaux produits au Canada et soumis au Journal canadien d'anesthésie a graduellement diminué au cours de la dernière décennie. Au sud de la frontière, environ $60 \%$ des manuscrits reçus l'année dernière par le journal Anesthesiology, provenaient de l'extérieur des ÉtatsUnis. Le Japon, l'Allemagne et la France ont actuellement les taux les plus élevés de soumission internationale d'articles, le Canada suivant derrière. ${ }^{4}$ L'inscription dans les programmes de recherche indique également que l'anesthésiologie reste à un niveau inférieur aux autres spécialités (Figure). En 1995, le Collège Royal des médecins et chirurgiens du Canada a mis sur pied le Programme de formation de clinicienschercheurs (PFC) pour faciliter la formation des cliniciens à la recherche (http://rcpsc.medical.org). Ce programme fournit une période intense de formation à la recherche d'un minimum de deux ans, pour un petit groupe de résidents qui sont choisis pendant les premières années de formation clinique de la résidence. En 2001, un seul diplômé parmi ceux des 16 programmes de résidence des universités canadiennes profitait de cette formidable occasion. ${ }^{5}$ Aussi, le nombre de sub- 
Stagiaires du PCC par spécialité ou sous-spécialité (2001)

\begin{tabular}{llll}
\hline & $u$ PCC & $\begin{array}{l}\text { Nombre } \\
\text { de résidents }\end{array}$ & $\%$ \\
\hline Chirurgie & 45 & 229 & 19,7 \\
Médecine & 28 & 282 & 9,9 \\
Pédiatrie & 8 & 91 & 8,8 \\
Psychiatrie & 6 & 108 & 5,6 \\
Ophtalmologie & 4 & 21 & 19,0 \\
Médecine communautaire & 4 & 18 & 22,2 \\
Pharmacologie clinique & 2 & 2 & 100,0 \\
Radio-oncologie & 1 & 10 & 10,0 \\
Gynécologie et obstétrique & 1 & 42 & 2,4 \\
Anatomopathologie & 1 & 13 & 7,7 \\
Anesthésiologie & 1 & 66 & 1,5 \\
\hline
\end{tabular}

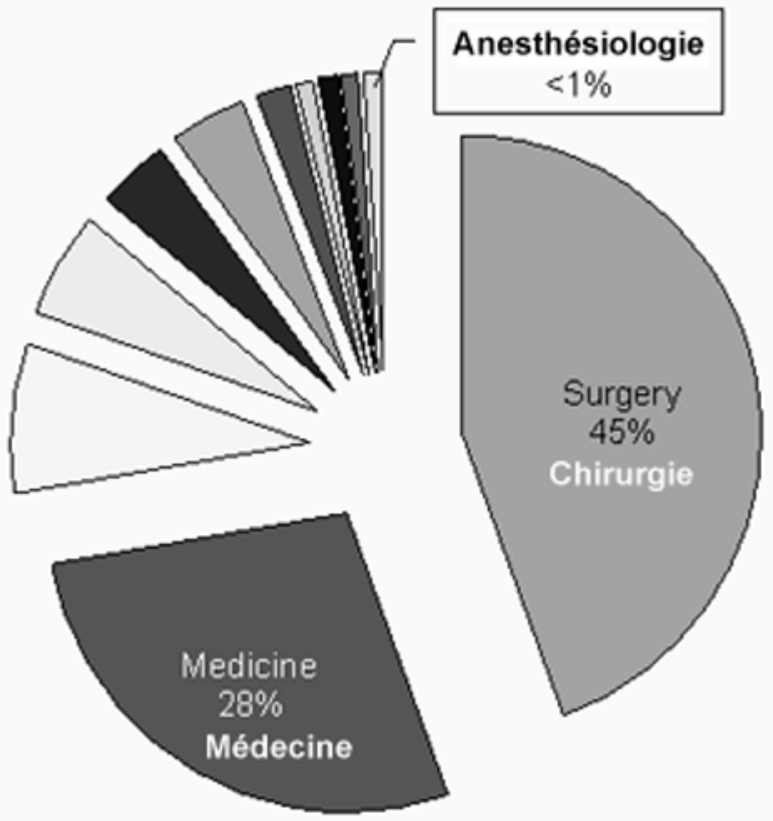

FIGURE Le nombre total de stagiaires du Programme de cliniciens chercheurs (PCC) en 2001 à l'université de Toronto comparé au nombre de résidents inscrits aux différentes disciplines médicales et chirurgicales en 2001 selon le rapport du Collège royal des médecins et chirurgiens du Canada. Aucun autre stagiaire du PCC n'a été inscrit dans d'autres programmes de résidence canadiens en anesthésie.

ventions de recherche allouées par les Instituts de recherche en santé du Canada (IRSC; http://www.cihr.ca) aux professeurs des Services d'anesthésie des universités canadiennes est significativement plus bas que celui de la plupart des autres spécialités médicales. Malgré une augmentation importante des possibilités de financement offertes par les IRSC, quelques anesthésiologistes seulement détiennent des subventions de recherche de cet organisme qui est notre principale agence de financement nationale pour la recherche en santé.

\section{Nouvelles possibilités de financement au Canada}

En dépit de ces sombres indicateurs, il y a de fortes raisons d'être optimiste. D'abord, le soutien à la recherche reliée à l'anesthésie est de plus en plus disponible au Canada. Le financement par la Fondation canadienne de recherche en anesthésie (FCRA) a augmenté pour la première fois depuis cinq ans grâce à une croissance significative du capital. Si on ajoute les augmentations de financement provenant des partenaires de l'industrie et des IRSC, les possibilités de financement offertes aux membres de la SCA ont atteint un sommet sans précédent. Les subventions de recherche de la SCA et du Programme de bourses de recherche comprennent maintenant une Bourse de carrière en recherche de $120000 \$$, une Bourse de recherche de 95000 \$ et six allocations de recherche totalisant $120000 \$$. De plus, ces possibilités de recherche reconnaissent différents niveaux d'expérience pour les nouveaux chercheurs et les chercheurs établis. La récente compétition pour obtenir la nouvelle Bourse de carrière en recherche sur l'anesthésie a été un immense succès, puisqu'elle compte 13 demandes d'inscription de qualité provenant de quatre provinces. Il y a certainement un grand intérêt pour la recherche au pays.

En plus de ces choix, de nouveaux partenariats entre la SCA et les IRSC sont présentement à l'étude pour créer un Clinician Scientists Career Pathway (Cheminement de carrière de clinicien chercheur). Le mandat des IRSC est de faciliter le transfert des connaissances (la recherche appliquée et la conversion de la recherche). L'acquisition de nouvelles connaissances médicales n'est plus suffisante; les IRSC cherchent plutôt des moyens novateurs d'accélérer le transfert du savoir pour améliorer les soins de santé. Nous croyons que les anesthésiologistes sont bien placés pour jouer ce rôle de formateur en physiologie, pharmacologie et médecine périopératoire. En nous concentrant sur nos forces et nos habiletés particulières, nous pouvons développer des programmes de recherche compétitifs et productifs.

Finalement, les départements d'anesthésie ont la possibilité de produire des ressources de recherche supplémentaires. Ainsi les chaires dotées, de recherche en anesthésie, fournissent généralement un soutien au salaire pour les chercheurs établis. Bien que les chaires dotées exigent des sommes considérables (habituelle- 
ment 2M\$), il en existe maintenant quelques-unes dont la chaire Harold Griffith à l'université McGill et la nouvelle chaire de recherche Fraser Elliott en anesthésie cardiovasculaire à l'université de Toronto. Aussi, le Laws Fellowship Award de l'université de Toronto assure un ou deux ans de soutien total au salaire des résidents en anesthésie ou aux boursiers diplômés entreprenant des études supérieures. Espérons que la prochaine décennie nous permette de voir la création de sources de financement additionnelles, étendues et stables à l'intérieur de la spécialité. Dans ce contexte, les plans de financement d'appoint (PFA) devraient donner l'occasion d'augmenter le temps réservé à la recherche. Actuellement, on retrouve des PFA à certains centres en Colombie Britannique, en Alberta et en Ontario. D'autres centres de santé universitaires songent à l'établissement de PFA ou sont déjà en négociation pour le faire; la recherche doit apparaître parmi les "priorités" de ces négociations.

Plus important encore, la composante de base d'un programme de recherche réussi est un chercheur bien formé appuyé par des guides compétents et une chaire universitaire engagée. Une brève période de formation à la recherche pendant une année de Fellowship n'est plus suffisante dans l'environnement compétitif d'aujourd'hui. Les candidats subventionnés par les IRSC ont en général deux ans au moins de formation à la recherche. La qualité du programme de formation ne doit toutefois pas être mésestimée. Pour la majorité des candidats, cela veut dire obtenir un diplôme à l'extérieur de leur discipline médicale. En choisissant un nombre limité de résidents ou de chercheurs déterminés, nous aussi pourrons augmenter cette cohorte à l'intérieur de notre spécialité. Les réunions de la SCA semblent un forum approprié pour les stagiaires du PCC, et pour d'autres, de discuter de leur expérience avec des candidats potentiels.

\section{Conclusion}

Il n'y a pas qu'une solution à la promotion de la productivité en recherche anesthésique. Cependant, au cours des dernières années, un nombre croissant de bourses et de mécanismes de financement de la recherche sont devenus disponibles. Le PCC et la restructuration des IRSC offrent un chance de réviser nos modèles actuels de formation et de soutien aux chercheurs. Nos Centres universitaire de sciences de la santé, la SCA et les agences de financement provinciales et nationales offrent maintenant de multiples chances de faire de la recherche en anesthésie. Notre défi est sans contredit de saisir l'occasion. Pour de nombreuses raisons, l'avenir de la recherche en anesthésie au Canada n'a jamais été aussi prometteur.

\section{Remerciements}

Les auteurs remercient les Dr David Bevan et Robert Byrick pour leurs commentaires constructifs.

\section{References}

1 Kohn LT, Corrigan JM, Donaldson MS. To Err is Human: Building a Safer Health System. Washington: DC, National Academy Press, 20418IOM, 2000.

2 Shephard DAE. Watching closely those who sleep. Guidelines to the practice of anesthesia. Can J Anaesth 1993; S40: 83-100.

3 Thompson SA, Wafford K. Mechanism of action of general anaesthetics-new information from molecular pharmacology. Curr Opin Pharmacol 2001; 1: 78-83.

4 Todd MM. 2002 Looks to be a banner year. American Society of Anesthesiologists Newsletter. Anesthesiology 2002.

5 Correspondence from the Royal College of Physicians and Surgeons of Canada. 